Research Article

\title{
Experimental Effects of Exercise on Forgetting
}

Lauren Ferguson, Justin Cantrelle, Paul Loprinzi *

Exercise \& Memory Laboratory, Department of Health, Exercise Science and Recreation Management, The University of Mississippi, University, MS 38677, USA; E-Mails: lefergus@go.olemiss.edu; ircantr1@go.olemiss.edu; pdloprin@olemiss.edu

* Correspondence: Paul Loprinzi; E-Mail: pdloprin@olemiss.edu

Academic Editor: Gerhard Litscher

Special Issue: Research of Exercise and Cognitive Function

OBM Integrative and Complementary Medicine

2018, volume 3 , issue 4

doi:10.21926/obm.icm.1804034
Received: September 6, 2018

Accepted: December 12, 2018

Published: December 17, 2018

\begin{abstract}
:
Background: The present study evaluates the effects of selective directed forgetting (SDF) and directed forgetting (DF) on subsequent memory retrieval and investigate whether acute exercise can facilitate the effects of SDF.
\end{abstract}

Methods: A four-arm ( $n=20$ per group; $N=80 ; M_{a g e}=20$ yrs), parallel-group, randomized controlled experiment was employed. The four groups included: 1) exercise plus SDF (Ex+SDF), 2) SDF only (SDF), 3) DF only (DF) and 4) $R$ (remember) only (R). Participants viewed two separate word lists (list 1 and list 2; L1 and L2). Based on group assignment, participants were directed to either forget, selectively forget, or remember List 1 . Outcome parameters included the number of words recalled from L1 and L2. Generally, participants directed to forget $L 1$ tend to recall more words from L2. Thus, L1 represents forgetting whereas $L 2$ represents the degree of memory enhancement. The exercise protocol involved a 15-minute bout of moderate-intensity treadmill exercise.

Results: We observed a significant main effect for $L 1$ Item-Type $\left(F=18.0, P<.001, \eta_{p}^{2}=.19\right)$, a significant main effect for $L 2$ Item-Type $\left(F=22.5, P<.001, \eta_{p}^{2}=.23\right)$, a significant $L 1$ Item-Type $x$ Cue interaction $\left(F=4.02, P=.01, \eta_{p}^{2}=.13\right)$, a marginally significant $L 2$ Item-Type $x$ Cue interaction $\left(\mathrm{F}=2.73, \mathrm{P}=.05, \mathrm{\eta}_{\mathrm{p}}^{2}=.10\right)$, a non-significant $\mathrm{L} 1$ Item-Type $\times$ L2 Item-Type

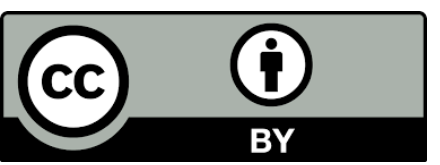

(C) 2018 by the author. This is an open access article distributed under the conditions of the Creative Commons by Attribution License, which permits unrestricted use, distribution, and reproduction in any medium or format, provided the original work is correctly cited. 
interaction ( $\left.\mathrm{F}=0.36, \mathrm{P}=.55, \mathrm{\eta}_{\mathrm{p}} \mathrm{p}^{\mathrm{a}} .005\right)$, and a marginally significant $\mathrm{L} 1$ Item-Type $\mathrm{x}$ L2 ItemType $x$ Cue interaction $\left(F=2.58, P=.06, \eta_{p}^{2}=.09\right)$. Directed forgetting facilitated forgetting, as the $D F(M=1.0)$ group recalled fewer words than the $R(M=2.10)$ group $(P=0.006)$. SDF alone was not effective in facilitating L1 forgetting or L2 enhancement. That is, recall (L1 or L2) was not significantly different in SDF compared to R. Acute exercise did not facilitate a forgetting effect, as EX + SDF was not different than SDF.

Conclusions: Our results provide evidence of a DF effect, but not SDF. Further, we did not observe any effects of acute exercise in facilitating SDF.

\section{Keywords}

Cognition; episodic memory; executive control; executive function; physical activity

\section{Introduction}

Episodic memory, part of the declarative memory system, refers to the retrospective recall of an event/episodic, evaluated in a spatio-temporal context [1]. Forgetting refers to the failure to either recall or retain information into present consciousness, and may be influenced from the decay of memories over time, accumulation of similar interfering experiences, and changes in the physical context that impair retrieval processes [2]. The ability to actively forget unwanted memories may facilitate learning and retrieval of new memories [3], which may be influenced by complex inhibitory cognitions such as attentional switching, active inhibition, and working memory [4], all of which may be favorably influenced by acute moderate-intensity exercise [5-8].

A typical paradigm addressing directed forgetting is to have participants, for example, read a list of words (List 1) and then afterward, one group is told to forget the words from List 1. Participants directed to forget List 1 tend to recall more words from List 2 [9]. Unlike directed forgetting, selective directed forgetting (SDF) has been less investigated and has produced mixed findings [10-19]. Selective directed forgetting involves, for example, receiving a cue to forget a subset of items from List 1. For example, after being exposed to 10 words from List 1 ( 5 in red font and 5 in green font), participants are instructed to forget the 5 words displayed in red font.

The literature demonstrates inconsistent findings regarding the effectiveness of SDF [10-17]. For example, some research demonstrates that when different to-be-remembered stimuli can be thematically integrated, directed forgetting does not occur, but when the stimuli is unrelated and cannot be easily integrated, SDF may occur [11]. Other research, however, across variations in the stimuli and study population, repeatedly demonstrates that SDF does not occur [17]. Given these inconsistent findings, the present study further evaluated the effects of SDF on memory recall. Additionally, and yet to be investigated, we also explore whether acute exercise can facilitate the effects of SDF on memory recall. Previous research demonstrates that acute exercise can facilitate episodic memory [20-26], but no studies, to date, have examined whether acute exercise can facilitate SDF. Such an effect is plausible given the exercise-induced mechanisms on episodic memory and the cellular basis for directed forgetting. That is, pathways influencing memory formation and memory forgetting may be altered by acute exercise engagement. 
Recent work $[3,27]$ has discussed the cellular basis of directed forgetting, which appears to be distinct from the cellular and molecular mechanisms influencing memory formation and persistence. In brief, neurobiological mechanisms of forgetting involves passive and active forgetting. Passive forgetting is postulated to occur through the 1) loss of contextual cues across time that impairs the retrieval of a memory; 2) interference during retrieval from other similar memories accumulated over time (e.g., forgetting where the car is parked because of having previously parked in that lot many times in the past); and 3) the natural decay of the memory trace [27]. Active forgetting is postulated to occur from 1) interference-based stimuli when the competing information occurs before (proactive interference) or after (retroactive) the learning event; 2) retrieval-induced forgetting, indicating that when certain aspects of a memory are retrieved, this may suppress the recall of other aspects related to the recalled memory; 3 ) motivated forgetting, occurring when cognitive mechanisms are engaged to weaken a memory trace (perhaps due to an unpleasant quality of the memory); and 4) intrinsic forgetting, which involves the ability of "forgetting cells" to degrade biochemical and molecular pathways of the memory trace. Regarding the latter, the protein, Rac1, a member of the Rho GTPase family, appears to be a key protein involved in intrinsic forgetting, and specifically, the Dopamine/Rac1/Cofilin signaling pathway [27]. That is, the binding of dopamine to the DAMB receptor activates the Rac1-Cofilin pathway, which negatively influences actin remodeling (prevents actin reassembly; actin is a key protein involved in modulating the shape and structure of dendritic spines), and in turn, decreases synaptic/dendritic size and growth.

Although previous work provides mixed findings regarding the effectiveness of SDF [10-17], emerging work suggests that executive control may play a critical role in memory suppression [28]. Fixating on a different task or target may help to enhance diversionary associations, and in turn, suppress the memory, as well as prevent independent cues from activating the unwanted target or memory [28]. This may be under direct influence via executive control, inhibitory-based cognitive mechanisms, which has been repeatedly shown to be favorably influenced by acute moderate-intensity exercise $[29,30]$. Thus, we anticipate that the main mechanism through which exercise may facilitate SDF is through exercise-induced alterations in executive control [31]. Recent work provides support for this assertion. For example, acute exercise has been shown to increase P3 amplitude and reduce N450 amplitude, key neural markers underlying improved executive control [32].

Couched within the above, the purpose of this study was to evaluate the effects of SDF on subsequent memory retrieval and investigate whether exercise can facilitate the effects of SDF. Similar to previous work, we hypothesize that directed forgetting will have a list 1 forgetting and list 2 enhancement effect, but we anticipate that SDF in isolation will not be effective in list 1 forgetting and list 2 enhancement. However, we hypothesize that exercise coupled with SDF will induce list 1 forgetting and list 2 enhancement.

\section{Materials and Methods}

\subsection{Study Design}

A four-arm, parallel-group randomized controlled intervention was employed. Participants were randomized into one of four groups. One of the experimental groups engaged in a brisk 
treadmill walk for 15 minutes, while the other three groups did not engage in an exercise bout. The four groups included: 1) exercise plus SDF (Ex+SDF), 2) SDF only (SDF), 3) DF (directed forgetting) only (DF) and 4) $R$ (remember) only (R). Details on the procedures of these groups are explained in the protocol below.

\subsection{Participants}

Each group included 20 participants ( $N=80 ; n=20$ per group) [20-23]. Recruitment occurred via a convenience-based, non-probability sampling approach (classroom announcement and word-ofmouth). Participants included undergraduate and graduate students between the ages of 18 and 35 yrs.

Additionally, and similar to other studies [33-36], participants were excluded if they:

Self-reported as a daily smoker [37, 38]

Self-reported being pregnant [39]

Exercised within 5 hours of testing [40]

Consumed caffeine within 3 hours of testing [41]

Had a concussion or head trauma within the past 30 days [42]

Took marijuana or other illegal drugs within the past 30 days [43]

Were considered a daily alcohol user (>30 drinks/month for women; >60 drinks/month for men) [44]

\subsection{Exercise Protocol}

Those randomized to the exercise group (Ex+SDF) walked on a treadmill for 15 minutes at a self-selected "brisk walking pace" (minimum speed of $3.0 \mathrm{mph}$ ), a pace as if they were "late for catching the bus." This protocol has previously been shown to enhance episodic memory function [45]. This bout of exercise occurred prior to the presentation of the first word list. Participants rested (sat) for 5 minutes after the bout of exercise before being presented with the first word list (details explained below).

\subsection{Non-Exercise Protocol}

As stated above, the Ex+SDF group engaged in an acute bout of exercise for 15-minutes and then rested for 5-minutes before starting the memory protocol. The three other groups (SDF, DF, and R) engaged in a seated time-matched 20-minute task before starting the memory protocol. This involved playing a medium-level, on-line administered, Sudoku puzzle. The website for this puzzle is located here: https://www.websudoku.com/.

\subsection{Memory Assessment}

The SDF involved the presentation of two separate word lists (list 1 and list 2; L1 and L2). Each list consisted of 16 unrelated words from the Toronto Word Pool. The order of words on each list were randomly assigned.

Half of the words from each list were in red color and the other half were in green color, which were randomly assigned. The presentation order of the red and green words was randomized, but 
no more than two words of the same color followed each other. Each word was presented on a computer screen at a rate of 4 seconds, followed by a 1-second inter-stimulus interval.

Following L1 presentation, participants received one of three types of instructions. Those randomized into the SDF condition were given the following instructions: "You now will be exposed to a different list of words in which you will be asked to recall later in this experiment. Importantly, please forget the red words from the previous list that you just saw. You will not be asked to remember any of the red words from that previous list, and it is important to try and forget those previous red words, as forgetting those words will help you remember the green words from the previous task as well as words from the upcoming task."

Those randomized into the DF (directed forgetting) group were told: "You now will be exposed to a different list of words in which you will be asked to recall later in this experiment. Importantly, please forget all the words from the previous list that you just saw. You will not be asked to remember any of these words from that previous list, and it is important to try and forget all those words, as forgetting those words will help you remember the words from the next task."

Those randomized into the $\mathrm{R}$ (remember) group were told: "You will now be exposed to a different list of words in which you will be asked to recall later in this experiment. Please also do your best to remember all the words from the list you were just exposed to, as you will be asked to recall words from both lists."

After the presentation of $\mathrm{L2}$, for 30-seconds, participants completed simple arithmetic problems. Following this, participants were told: "You now will recall as many words as possible. Please do not worry about the color of any of the words. You now have 90-seconds to write down as many words as you can remember from L1." After this 90-second period, they were told: "You now will recall as many words as possible from L2. Please do not worry about the color of any of the words. You now have 90-seconds to write down as many words as you can remember from L2." Notably, the order of L1 and L2 were counterbalanced, as half of the participants recalled L1 first, whereas the other half recalled $L 2$ first.

\subsection{Additional Assessments}

Additional assessments were completed (at the beginning of the visit) to ensure that conditions were similar on these parameters. To assess mood status, which may influence memory capacity [46], participants completed the Positive and Negative Affect Schedule (PANAS) [47]. For this mood survey, participants rated 20 items (e.g., excited, upset, irritable, attentive) on a Likert scale (1, very slightly or not at all; to 5, extremely), with half of the items constituting a "positive" mood state, with the other half being a "negative" mood state. To provide anthropometric characteristics of the sample, body mass index was calculated from measured height and weight. Further, waist circumference was assessed using a tape measure. Lastly, before, during and after the exercise and control conditions, heart rate (chest-strapped Polar monitor, F1 model) was assessed.

\subsection{Statistical Analysis}

All statistical analyses were computed in SPSS (v. 24). A $2 \times 2 \times 4$ mixed analysis of variance was computed. This involved a 2 (L1 Item-Type) x 2 (L2 Item-Type) x 4 (Cue) model. Cue (EX+SDF, SDF, $D F, R$ ) served as the between-subjects factor, and L1 Item-Type (recall of red items, recall of green 
items) and L2 Item-Type (recall of red items, recall of green items) served as the within-subject factors. Statistical significance was established as an alpha of 0.05. Partial eta-squared $\left(\eta^{2}\right.$ p $)$ values are provided as measures of effect size.

\section{Results}

Table 1 displays the characteristics of the participants among the 4 groups. Across all 4 groups, participants were similar regarding age, gender, race-ethnicity, weight status, psychological mood state at the beginning of the visit, and resting heart rate. As expected, the heart rate was substantively different in the EX+SDF group compared to the other groups (all $P^{\prime} s<0.05$ ). The 3 non-exercise groups had heart rates that ranged from $75-85 \mathrm{bpm}$ for the 20 -min control period prior to the memory task. For the EX+SDF group, heart rate increased up to approximately 130 bpm during the bout of exercise.

Table 1 Characteristics (point estimate, SD) of the study variables.

\begin{tabular}{lllll}
\hline Variable & EX + SDF & SDF & DF & $\mathbf{R}$ \\
\hline $\mathbf{N}$ & 20 & 20 & 20 & 20 \\
Age (mean years) & $20.2(1.0)$ & $20.5(1.0)$ & $20.9(2.0)$ & $20.8(1.1)$ \\
\% Female & 75.0 & 75.0 & 80.0 & 75.0 \\
Race-Ethnicity (\%) & & & & \\
White & 90.0 & 90.0 & 85.0 & 90.0 \\
Black & 10.0 & 10.0 & 10.0 & 5.0 \\
Other & 0.0 & 0.0 & 5.5 & 5.0 \\
BMI (mean kg/m ${ }^{2}$ ) & $22.6(2.8)$ & $23.8(3.3)$ & $24.6(4.2)$ & $25.2(4.0)$ \\
Waist circumference (mean cm) & $79.7(7.6)$ & $82.7(9.2)$ & $85.6(11.2)$ & $84.9(9.0)$ \\
Mood State & & & & \\
Positive, mean & $30.1(9.3)$ & $28.5(8.7)$ & $28.0(6.0)$ & $27.3(7.4)$ \\
Negative, mean & $11.2(1.6)$ & $12.3(3.0)$ & $12.3(2.7)$ & $12.0(2.8)$ \\
Exercise Parameters & & & & \\
Resting Heart Rate, mean bpm & $79.6(15.7)$ & $83.3(12.0)$ & $79.6(17.9)$ & $75.9(11.7)$ \\
Mid-Heart Rate, mean bpm & $125.8(15.2)$ & $84.1(14.0)$ & $77.3(17.8)$ & $77.0(9.0)$ \\
End-Heart Rate, mean bpm & $129.5(16.7)$ & $83.2(14.6)$ & $77.6(17.9)$ & $75.9(10.1)$ \\
Post-Heart Rate, mean bpm & $83.5(13.7)$ & $81.4(11.4)$ & $77.8(16.9)$ & $73.5(7.7)$ \\
Treadmill Speed, mean mph & $3.8(0.2)$ & - & - & - \\
\hline
\end{tabular}

Table 2 displays the memory scores across the 4 groups. For L1, the EX+SDF, SDF, and DF groups recalled fewer items than $\mathrm{R}$, for both red and green items. For $\mathrm{L} 2$, DF recalled more items than $\mathrm{R}$ for red words, and for green words, EX+SDF, SDF, and DF recalled more items than $\mathrm{R}$. Regarding the $2 \times 2 \times 4$ mixed analysis of variance model, there was a significant main effect for L1 Item-Type $\left(F=18.0, P<.001, \eta_{p}^{2}=.19\right)$, a significant main effect for $L 2$ Item-Type $(F=22.5, P<.001$, $\left.\eta_{p}^{2}=.23\right)$, a significant $L 1$ Item-Type $x$ Cue interaction $\left(F=4.02, P=.01, \eta_{p}^{2}=.13\right)$, a marginally significant $L 2$ Item-Type $x$ Cue interaction ( $\left.F=2.73, P=.05, \eta_{p}^{2}=.10\right)$, a non-significant L1 Item-Type $x$ 
$\mathrm{L} 2$ Item-Type interaction ( $\left.\mathrm{F}=0.36, \mathrm{P}=.55, \mathrm{\eta}_{\mathrm{p}}^{2}=.005\right)$, and a marginally significant $\mathrm{L} 1$ Item-Type $\mathrm{x}$ L2 Item-Type $x$ Cue interaction $\left(F=2.58, P=.06, \eta_{p}^{2}=.09\right)$.

Table 2 Memory scores (mean, SD) across the 4 experimental groups.

\begin{tabular}{lllll}
\hline Variable & EX + SDF & SDF & DF & R \\
\hline List 1 & & & & \\
Correct number of red words & $1.25(0.78)$ & $1.00(1.12)$ & $0.50(0.51)$ & $1.35(0.93)$ \\
Correct number of green words & $2.00(1.25)$ & $1.70(0.97)$ & $1.00(0.91)$ & $2.10(1.44)$ \\
List 2 & & & & \\
Correct number of red words & $1.05(0.99)$ & $1.50(1.23)$ & $2.15(1.59)$ & $2.00(1.07)$ \\
Correct number of green words & $2.60(1.81)$ & $2.25(1.99)$ & $2.10(1.16)$ & $1.90(1.29)$ \\
\hline
\end{tabular}

Post-hoc tests were computed for the significant interaction models. For the significant L1 Item-Type $x$ Cue interaction $\left(F=4.02, P=.01, \eta_{p}^{2}=.13\right)$, for the red words, significant group differences occurred for $E X+\operatorname{SDF}(M=1.25)$ vs. $D F(M=0.5) \quad(P=0.001)$, and $R(M=1.35)$ vs. $D F$ $(M=0.5)(P=0.001)$. Similarly, for the green words, significant differences emerged for $E X+S D F$ $(M=2.0)$ vs. $D F(M=1.0)(P=0.006)$, SDF $(M=1.70)$ vs. $D F(M=1.0)(P=0.02)$, and $D F(M=1.0)$ vs. $R$ $(\mathrm{M}=2.10)(\mathrm{P}=0.006)$.

For the L2 Item-Type $x$ Cue interaction $\left(F=2.73, P=.05, \eta_{p}^{2}=.10\right)$ model, for the red words, significant group differences occurred for $E X+S D F(M=1.05)$ vs. $D F(M=2.15)(P=0.01)$, and $E X+$ $\operatorname{SDF}(M=1.05)$ vs. $R(M=2.0)(P=0.006)$. For the green words, there were no significant between group differences.

\section{Discussion}

In alignment with our hypothesis, DF facilitated forgetting, as the $D F(M=1.0)$ group recalled fewer words than the $R(M=2.10)$ group $(P=0.006)$. However, $D F$ did not facilitate $L 2$ enhancement. Also, in support of our hypothesis, SDF alone was not effective in facilitating L1 forgetting or L2 enhancement. That is, recall (L1 or L2) was not significantly different in SDF compared to R. However, in contrast to our hypothesis, acute exercise did not facilitate a forgetting effect, as EX + SDF was not different than SDF for either red or green words. Conclusions are also similar for L2 enhancement; SDF was not different than R, and EX + SDF was not different than SDF.

Our findings that DF may facilitate forgetting is in alignment with other related research, demonstrating that executive control-induced DF may help to facilitate the suppression of unwanted memories [28]. Our inability to demonstrate a selective effect on forgetting also aligns with other work [17]. Further, some research demonstrates that attempting to selectively forget parts of the memory may detrimentally influence the recall of other aspects of the memory [15].

Counter to our hypothesis, acute exercise did not facilitate an SDF effect. We anticipated it would as exercise, particularly moderate-intensity exercise, has been shown to enhance executive control [29, 30], which may be a mechanism facilitating directed forgetting [28]. However, regarding the latter, executive control may play a more important role in DF as opposed to SDF [28]. Thus, future work in this novel line of inquiry should consider evaluating whether acute 
exercise can effectuate DF. The intensity and timing of exercise may also play a moderating role [48], which should be carefully considered in future work on this topic.

A limitation of this study is the relatively small homogenous sample, which limits our study's generalizability and statistical power. Further, although no participants subjectively reported color impairment, participants were not objectively screened for visual color impairment. Additionally, the previous demonstrations of a possible SDF effect employed sentence-type assessments, as opposed to our word-list approach. Thus, future work on this topic may wish to consider a sentence-type memory protocol when evaluating the effects of exercise on SDF. Participants in the present study recalled more green words over red words, and since the to-be-forgotten color was not counterbalanced, this may have induced a general bias toward green words. This, along with considering output order or clustering [49], should be considered in future work on this topic. Several strengths of this study include the novel examination and experimental design.

In conclusion, the present experiment aimed to evaluate the effects of DF and SDF on memory, as well as whether acute moderate-intensity exercise can facilitate SDF. Our results provide evidence of a DF effect, but not SDF. Further, we did not observe any effects of acute exercise in facilitating SDF.

\section{Acknowledgments}

We have no conflicts of interest and no funding was used to prepare this manuscript.

\section{Author Contributions}

LF and JC were involved in data collection and manuscript feedback. PDL was involved in study conceptualization, data analyses and manuscript writing.

\section{Competing Interests}

The authors have declared that no competing interests exist.

\section{References}

1. Tulving E. Episodic memory: from mind to brain. Annu Rev Psychol. 2002; 53: 1-25.

2. Anderson MC, Hanslmayr S. Neural mechanisms of motivated forgetting. Trends Cogn Sci. 2014; 18: 279-292.

3. Richards BA, Frankland PW. The persistence and transience of memory. Neuron. 2017; 94 : 1071-1084.

4. Gamboa OL, Sung Lai Yuen K, von Wegner F, Behrens M, Steinmetz H. The challenge of forgetting: Neurobiological mechanisms of auditory directed forgetting. Hum Brain Mapp. 2018; 39: 249-263.

5. Chang YK, Labban JD, Gapin JI, Etnier JL. The effects of acute exercise on cognitive performance: A meta-analysis. Brain Res. 2012; 1453: 87-101.

6. McMorris T. Developing the catecholamines hypothesis for the acute exercise-cognition interaction in humans: Lessons from animal studies. Physiol Behav. 2016; 165: 291-299.

7. McMorris T, Turner A, Hale BJ, Sproule J. Exercise-cognition interaction: Neuroscience perspectives. Cambridge: Academic Press; 2016. p. 65-103. 
8. McMorris T, Sproule J, Turner A, Hale BJ. Acute, intermediate intensity exercise, and speed and accuracy in working memory tasks: A meta-analytical comparison of effects. Physiol Behav. 2011; 102: 421-428.

9. MacLeod CM. Intentional forgetting: Interdisciplinary approaches. Mahwah: Lawrence Erlbaum Associates; 1998.

10. Aguirre C, Gomez-Ariza CJ, Bajo MT, Andres P, Mazzoni G. Selective voluntary forgetting in young and older adults. Psychol Aging. 2014; 29: 128-139.

11. Delaney PF, Nghiem KN, Waldum ER. The selective directed forgetting effect: can people forget only part of a text?. Q J Exp Psychol. 2009; 62: 1542-1550.

12. Gomez-Ariza CJ, Iglesias-Parro S, Garcia-Lopez L, Diaz-Castela MM, Espinosa-Fernandez L, Muela JA. Selective intentional forgetting in adolescents with social anxiety disorder. Psychiatry Res. 2013; 208: 151-155.

13. Kliegl O, Pastotter B, Bauml KH. List-method directed forgetting can be selective: evidence from the 3-list and the 2-list tasks. Mem Cogn. 2013; 41: 452-464.

14. Pastotter B, Kliegl O, Bauml KH. List-method directed forgetting: Evidence for the reset-ofencoding hypothesis employing item-recognition testing. Memory. 2016; 24: 63-74.

15. Sahakyan L. Destructive effects of "forget" instructions. Psychon Bull Rev. 2004; 11: 555-559.

16. Storm BC, Koppel RH, Wilson BM. Selective cues to forget can fail to cause forgetting. Q J Exp Psychol. 2013; 66: 29-36.

17. Akan M, Sahakyan L. Repeated failures to obtain selective directed forgetting in lab and online samples and variations in stimuli. Memory. 2017; 26: 1-12.

18. Pastotter B, Kliegl O, Bauml KH. List-method directed forgetting: The forget cue improves both encoding and retrieval of postcue information. Mem Cogn. 2012; 40: 861-873.

19. Foster NL, Sahakyan L. The role of forget-cue salience in list-method directed forgetting. Memory. 2011; 19: 110-117.

20. Loprinzi PD, Kane CJ. Exercise and cognitive function: a randomized controlled trial examining acute exercise and free-living physical activity and sedentary effects. Mayo Clin Proc. 2015; 90: 450-460.

21. Crush EA, Loprinzi PD. Dose-response effects of exercise duration and recovery on cognitive functioning. Percept Mot Skills. 2017; 124: 1164-1193.

22. Frith E, Sng E, Loprinzi PD. Randomized controlled trial evaluating the temporal effects of high-intensity exercise on learning, short-term and long-term memory, and prospective memory. Eur J Neurosci. 2017; 46: 2557-2564.

23. Sng E, Frith E, Loprinzi PD. Temporal effects of acute walking exercise on learning and memory. Am J Health Promot. 2017; 32: 1518-1525.

24. Loprinzi PD, Frith E, Edwards MK, Sng E, Ashpole N. The effects of exercise on memory function among young- to middle-age adults: Systematic review and recommendations for future research. Am J Health Promot. 2017; 32: 691-704.

25. Loprinzi PD, Edwards MK, Frith E. Potential avenues for exercise to activate episodic memoryrelated pathways: a narrative review. Eur J Neurosci. 2017; 46: 2067-2077.

26. Loprinzi PD, Edwards MK. Exercise and implicit memory: A brief systematic review. Psychol Rep. 2017; 121: 1072-1085.

27. Davis RL, Zhong Y. The biology of forgetting-A perspective. Neuron. 2017; 95: 490-503. 
28. Anderson MC, Green C. Suppressing unwanted memories by executive control. Nature. 2001; 410: 366-369.

29. Hillman $\mathrm{CH}$, Snook EM, Jerome GJ. Acute cardiovascular exercise and executive control function. Int J Psychophysiol. 2003; 48: 307-314.

30. Ludyga S, Gerber M, Brand S, Holsboer-Trachsler E, Puhse U. Acute effects of moderate aerobic exercise on specific aspects of executive function in different age and fitness groups: A meta-analysis. Psychophysiology. 2016; 53: 1611-1626.

31. Mullen SP, Hall PA. Editorial: Physical activity, self-regulation, and executive control across the lifespan. Front Hum Neurosci. 2015; 9: 614.

32. Hsieh SS, Huang CJ, Wu CT, Chang YK, Hung TM. Acute exercise facilitates the N450 Inhibition Marker and P3 Attention Marker during stroop test in young and older adults. J Clin Med. 2018; 7: DOI: 10.3390/jcm7110391

33. Yanes D, Loprinzi PD. Experimental effects of acute exercise on iconic memory, short-term episodic, and long-term episodic memory. J Clin Med. 2018; 7: DOI: 10.3390/jcm7060146.

34. Siddiqui A, Loprinzi PD. Experimental investigation of the time course effects of acute exercise on false episodic memory. J Clin Med. 2018; 7: DOI: 10.3390/jcm7070157.

35. Haynes IV JT, Frith E, Sng E, Loprinzi PD. Experimental effects of acute exercise on episodic memory function: Considerations for the timing of exercise. Psychol Rep. 2018; DOI: 10.1177/0033294118786688.

36. Haynes IV JT, Loprinzi PD. Acute cardiovascular exercise on proactive memory interference. J Cogn Enhanc. 2018.

37. Jubelt LE, Barr RS, Goff DC, Logvinenko T, Weiss AP, Evins AE. Effects of transdermal nicotine on episodic memory in non-smokers with and without schizophrenia. Psychopharmacology (Berl). 2008; 199: 89-98.

38. Klaming R, Annese J, Veltman DJ, Comijs HC. Episodic memory function is affected by lifestyle factors: A 14-year follow-up study in an elderly population. Neuropsychol Dev Cogn B Aging Neuropsychol Cogn. 2017; 24: 528-542.

39. Henry JD, Rendell PG. A review of the impact of pregnancy on memory function. J Clin Exp Neuropsychol. 2007; 29: 793-803.

40. Labban JD, Etnier JL. Effects of acute exercise on long-term memory. Res Q Exerc Sport. 2011; 82: 712-721.

41. Sherman SM, Buckley TP, Baena E, Ryan L. Caffeine enhances memory performance in young adults during their non-optimal time of day. Front Psychol. 2016; 7: 1764.

42. Wammes JD, Good TJ, Fernandes MA. Autobiographical and episodic memory deficits in mild traumatic brain injury. Brain Cogn. 2017; 111: 112-126.

43. Hindocha C, Freeman TP, Xia JX, Shaban NDC, Curran HV. Acute memory and psychotomimetic effects of cannabis and tobacco both 'joint' and individually: A placebocontrolled trial. Psychol Med. 2017; 47: 2708-2719.

44. Le Berre AP, Fama R, Sullivan EV. Executive functions, memory, and social cognitive deficits and recovery in chronic alcoholism: A critical review to inform future research. Alcohol Clin Exp Res. 2017; 41: 1432-1443.

45. Sng E, Frith E, Loprinzi PD. Temporal effects of acute walking exercise on learning and memory function. Am J Health Promot. 2018; 32: 1518-1525. 
46. Storbeck J, Maswood R. Happiness increases verbal and spatial working memory capacity where sadness does not: Emotion, working memory and executive control. Cogn Emot. 2016; 30: 925-938.

47. Watson D, Clark LA, Tellegen A. Development and validation of brief measures of positive and negative affect: the PANAS scales. J Pers Soc Psychol. 1988; 54: 1063-1070.

48. Loprinzi PD. Intensity-specific effects of acute exercise on human memory function: considerations for the timing of exercise and the type of memory. Health Promot Perspect. 2018; 8: 255-262.

49. Bjork RA, Whitten WB. Recency-sensitive retrieval processes in long-term free recall. Cogn Psychol. 1974; 6: 173-189.

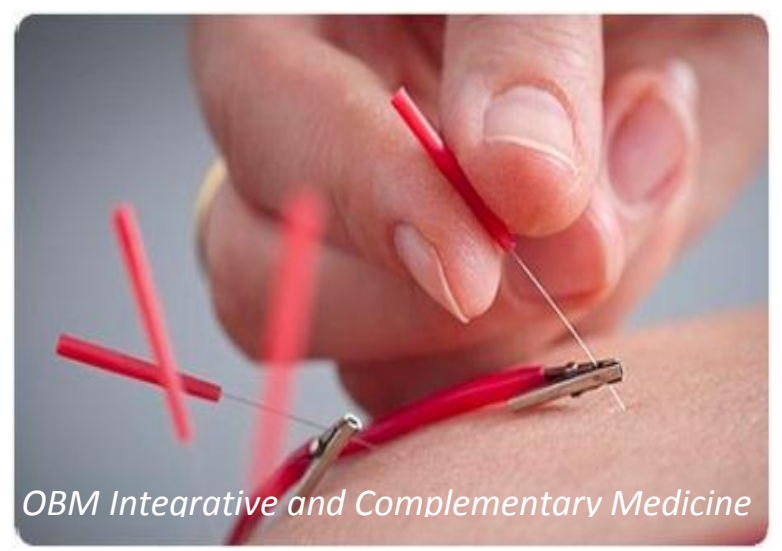

Enjoy OBM Integrative and Complementary Medicine by:

1. Submitting a manuscript

2. Joining in volunteer reviewer bank

3. Joining Editorial Board

4. Guest editing a special issue

For more details, please visit: http://www.lidsen.com/journals/icm 